\title{
Transfer of microwave energy along a filament plasma column in air B. Prade ${ }^{1}$, A. Houard ${ }^{1}$, J. Larour ${ }^{2}$, M. Pellet ${ }^{3}$ and A. Mysyrowicz ${ }^{1, a)}$
}

${ }^{1}$ LOA, ENSTA ParisTech, CNRS, Ecole polytechnique, Université Paris-Saclay, 828 bd des Maréchaux, 91762 Palaiseau cedex, France

${ }^{2}$ LPP, CNRS, Ecole polytechnique, UPMC Univ. Paris 06, Univ. Paris-Sud, Observatoire de Paris, Université Paris-Saclay, Sorbonne Universités, PSL Research University, 4 place Jussieu, 75252 Paris, France

${ }^{3}$ Ministère de la Défense, F-00457 Armées, France

${ }^{\text {a) Electronic mail: andre.mysyrowicz@ensta.fr. }}$

\begin{abstract}
We demonstrate the coupling of microwave radiation into a plasma channel formed by laser filamentation in air, leading to the amplification by two orders of magnitude of longitudinal oscillations of the plasma. Transfer of this longitudinal excitation towards unexcited region of the plasma column occurs over more than $10 \mathrm{~cm}$, in good agreement with a theoretical model describing the propagation of a $T M$ wave guided along the surface between air and plasma. We foresee that high power low frequency electromagnetic waves injected into a multi-filament plasma could initiate and sustain a long-lived plasma over several meters distance.
\end{abstract}

A sufficiently intense femtosecond laser pulse can propagate in air in the form of a filamentary pulse that maintains a high intensity over several hundreds of meters [1]. This non linear propagation regime results from a complex interplay between diffraction, the optical Kerr effect, high field ionization, plasma defocusing and other nonlinear effects. A column of weakly ionized plasma, of free electron density $n_{e} \sim 10^{17} \mathrm{~cm}^{-3}$, is left in the wake of the filamentary pulse. This plasma column can lead to many applications. It is the source of a forward oriented conical emission of pulsed $\mathrm{THz}$ radiation [2]. It can form a low-jitter fast spark gap [3]. It can also trigger and guide meter long electric discharges [4], allowing contactless transfer of large currents [5]. In turn, long-lived guided discharges can act as virtual antennas [6] or could improve the aerodynamics of fast moving object through atmosphere [7].

In this article we use an oscillating RF electric field to excite an electromagnetic $T M$ wave with maximum electric field amplitude located at the interface between a small section of a multi-filament plasma column and the non ionized external medium [8-9]. This surface wave propagates out of the excitation region, allowing the transfer, over a significant distance, of microwave energy to the plasma in the form of longitudinal oscillations. The propagation distance should reach several meters in the case of lower frequency electromagnetic waves. Guided propagation of microwave radiation by filaments using a different approach has been discussed previously both theoretically [10-12] and experimentally [13-15]. We suggest that a self-sustained plasma antenna could be realized if sufficient microwave or VHF power is injected.

Fig. 1 shows the experimental arrangement: the plasma channel is created by filamentation of intense laser pulses at $800 \mathrm{~nm}$. Such laser pulses with 200-mJ energy and adjustable duration between $50 \mathrm{fs}$ and 1 ps are delivered by a multiterawatt CPA laser source (Enstamobile) at a rate of $10 \mathrm{~Hz}$. When focused in air with a lens of 5-m focal length, they create a bundle of ionized filaments merging into a cylindrical plasma of average density $n_{e} \sim 10^{17} \mathrm{~cm}^{-3}$, roughly one millimeter in diameter and 1- 2 meter in length (see Fig. 2) [16]. A microwave field is delivered to a small section of the plasma using a well-known source called surfatron [17]. The surfatron consists of a commercial microwave generator (magnetron) emitting continuously at $2.45 \mathrm{GHz}$ with a maximum power of 500 Watt (GMP 12 KED by SAIREM S.A.). The microwave is injected as a $T M$ mode via a capacitive coupler in a 40-mm long, annular brass cavity surrounding the filament bundle, itself contained in a fused silica tube. Transfer of microwave to the plasma region occurs via a leak in the annular cavity, an oscillating longitudinal E-field being imposed across the launching gap. The diameter of the surfatron hole is 6 $\mathrm{mm}$.

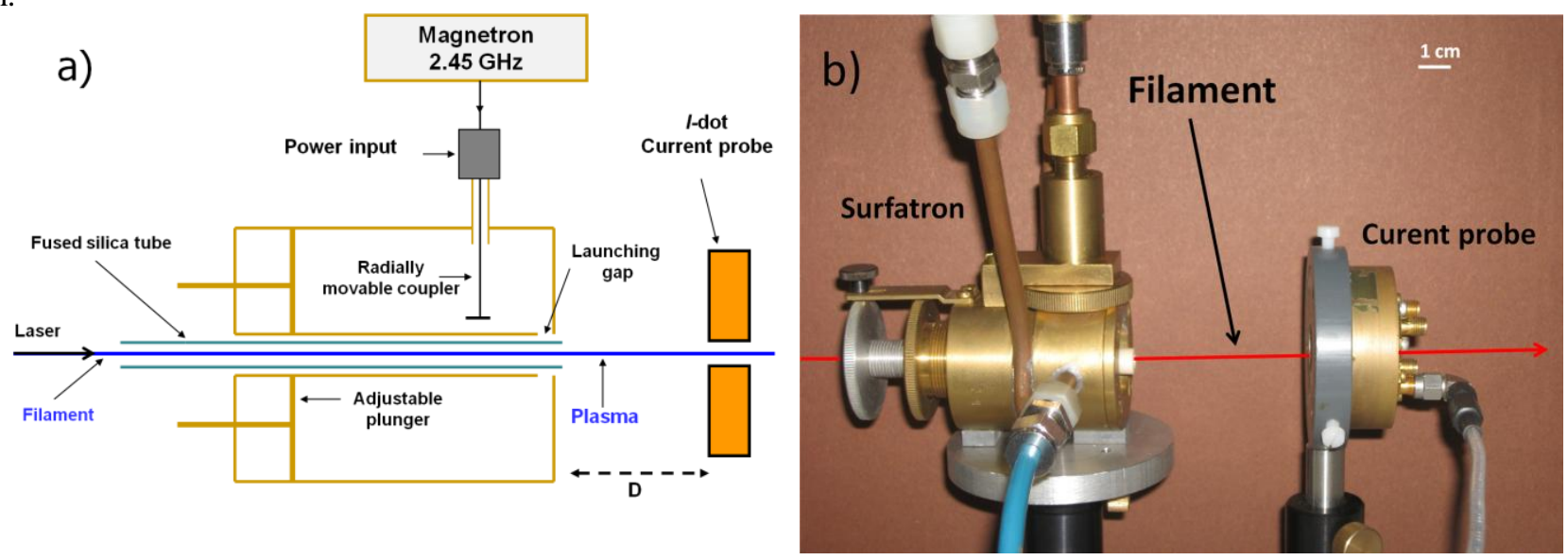

Fig. 1 (a) General set-up. (b) Photograph of the experiment. 


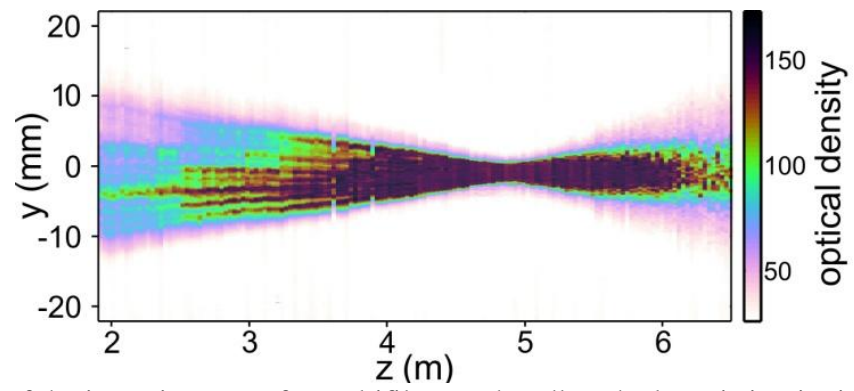

Fig. 2 Side view (yz, $x=0$ plane) of the intensity map of a multifilament bundle. The laser is impinging from the left. Dark colors (color scale > 100) correspond to ionized regions.

In our experiment, the microwave radiation was produced continuously while the laser operated in single pulse mode. No provision was made to synchronize the time of arrival of the laser pulse with a cycle of the microwave field. To measure the transfer of microwave energy to the filament plasma, we detected the induced longitudinal electronic currents in the plasma by using a fast $I$-dot probe placed outside the surfatron cavity, at a distance $D$. The $I$-dot probe allows the contactless measurement of longitudinal currents in the plasma [18]. It was connected with a shielded 50-ohm cable to an oscilloscope of $10-\mathrm{GHz}$ bandwidth. We checked that no signal was detected by the $I$-dot probe in the absence of laser filament, even at maximum microwave power.

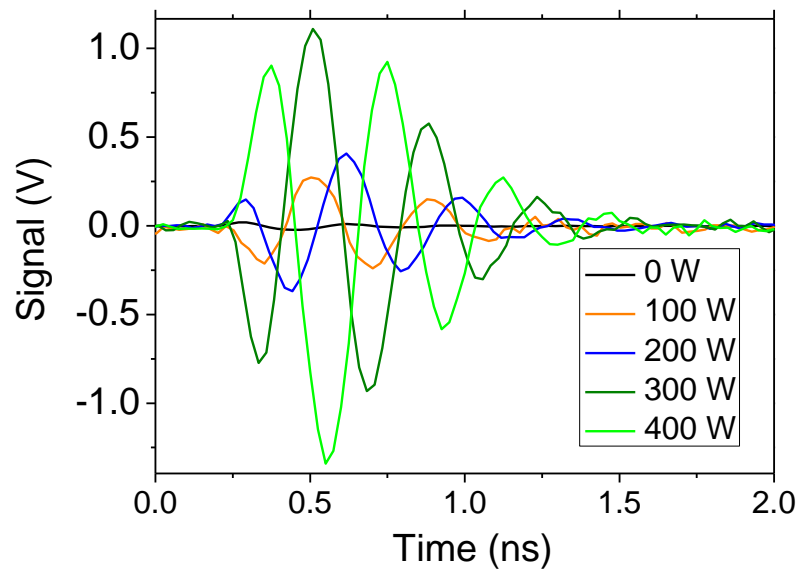

Fig. 3 Longitudinal current signal measured by the $I$-dot probe as a function of microwave power in the presence of filament. The probe is placed away from the surfatron exit $(D=6.3 \mathrm{~cm})$.

In Fig. 3, we show the signals registered in the presence of the filaments by the $I$-dot probe for different microwave powers at a fixed position $D=6.3 \mathrm{~cm}$. Since there is no synchronization between the laser pulse and the surfatron, different laser shots arrived randomly in the cavity at different phases of the microwave field. We have selected from a large number of shots the highest signals recorded for a given microwave value. They correspond to the arrival of the pulse at the positive or negative peak of a microwave cycle. The weak signal recorded in the absence of microwave (black curve) corresponds to the longitudinal oscillation that is imparted to the plasma during its formation, due to the ponderomotive force of the laser pulse [2].

We have measured the maximum signal detected by the $I$-dot probe as a function of distance $D$. The maximum signal divided by the maximum signal in the absence of microwave field is plotted in Fig. 4 for a microwave injected power of $P=$ $100 \mathrm{~W}$. It shows an amplification of the longitudinal current over a length longer than $15 \mathrm{~cm}$ beyond the exit of the surfatron. 


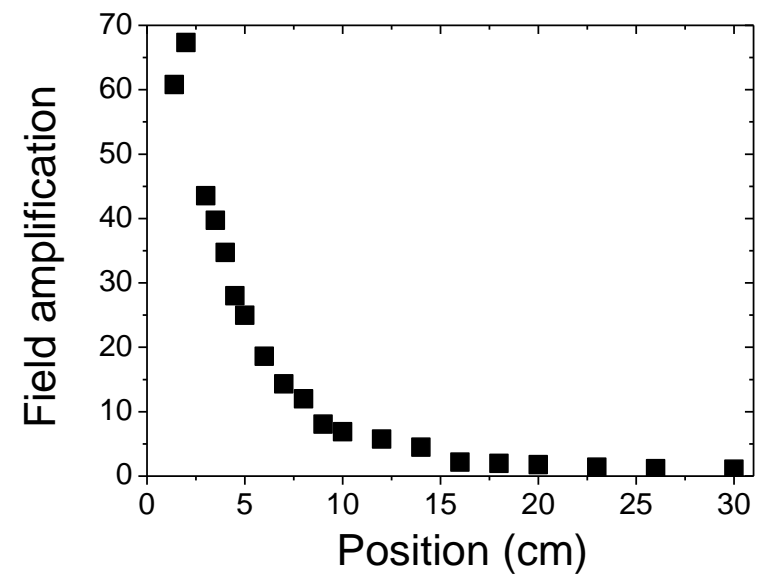

Fig. 4 Maximum signal measured by the $I$-dot probe divided by the maximum signal in the absence of microwave field as a function of the position along the filament measured for $P=100$ Watt.

To interpret the flow of excitation along the filament, we consider the propagation of a surface guided $T M$ wave. The filament is modeled as a homogeneous column of plasma of radius $r=a$ and complex susceptibility $\varepsilon_{p}$. The index of refraction of air and plasma are noted $n_{0}(r>a)$ and $n_{p}(r<a)$ respectively. For cold plasmas the index of refraction $n_{p}$ can be expressed by

$$
\varepsilon_{p}=n_{p}^{2}=1-\frac{\omega_{p}^{2} / \omega^{2}}{1-j v_{e} / \omega},
$$

where $\omega_{p}$ is the plasma circular frequency and $v_{e}$ is the frequency collision between electrons and neutral molecules.

$$
\omega_{p}=\sqrt{\frac{e^{2} n_{e}}{\varepsilon_{0} m_{e}}} .
$$

In the formulas $e$ is the charge of the electron, $m_{e}$ its mass, and $n_{e}$ is the electronic density of the plasma.

The field of a forward propagating mode can be written in the form:

$$
\left\{\begin{array}{l}
E(r, \theta) \exp (j \omega t) \exp \left(-j k n_{\text {eff }} z\right) \\
H(r, \theta) \exp (j \omega t) \exp \left(-j k n_{\text {eff }} z\right)
\end{array}\right.
$$

Here $\omega$ is the circular frequency of the microwave emitted by the surfatron, $k$ is the vacuum wave number, $n_{\text {eff }}$ is the complex effective index and $(r, \theta, z)$ are the coordinates in cylindrical coordinates, where the $Z$ axis is defined by the filament axis. Due to axial symmetry we restrict the solution to a $T M_{0}$ mode. By adopting non dimensional notations we let:

$$
W=k a \sqrt{n_{\text {eff }}^{2}-n_{0}^{2}} \text { and } U=k a \sqrt{n_{\text {eff }}^{2}-n_{p}^{2}} \text {. }
$$

With these notations the microwave field can be expressed for $r>a$ (in air)

$$
\begin{aligned}
& \left\{\begin{array}{l}
E_{r}^{0}(r, \theta)=j \frac{k a}{U} A n_{e f f} I_{1}\left(\frac{U r}{a}\right) \\
E_{\theta}^{0}=0 \\
E_{z}^{0}(r, \theta)=A I_{0}\left(\frac{U r}{a}\right)
\end{array},\right. \\
& \left\{\begin{array}{l}
H_{r}^{p}=H_{z}^{p}=0 \\
H_{\theta}^{p}(r, \theta)=j \frac{k a}{U} A n_{p}^{2} I_{1}\left(\frac{U r}{a}\right),
\end{array}\right.
\end{aligned}
$$

and for $r<a$ (inside the plasma) 


$$
\begin{aligned}
& \left\{\begin{array}{l}
E_{r}^{p}(r, \theta)=-j \frac{k a}{W} \frac{I_{0}(U)}{K_{0}(W)} A n_{e f f} K_{1}\left(\frac{W r}{a}\right) \\
E_{\theta}^{p}=0 \\
E_{z}^{p}(r, \theta)=A \frac{I_{0}(U)}{K_{0}(W)} K_{0}\left(\frac{W r}{a}\right)
\end{array}\right. \\
& \left\{\begin{array}{l}
H_{r}^{p}=H_{z}^{p}=0 \\
H_{\theta}^{p}(r, \theta)=-j \frac{k a}{W} \frac{I_{0}(U)}{K_{0}(W)} A n_{g}^{2} K_{1}\left(\frac{W r}{a}\right),
\end{array}\right.
\end{aligned}
$$

where $I$ and $K$ are the classical notations for Bessel functions and $A$ is a constant in $\mathrm{V} / \mathrm{m}$. Note that for simplicity in the expressions above $\mu_{0}$ does not appear so that $H$ and $E$ are both expressed in electric unities. The field continuity at the plasma boundary leads to the dispersion equation that reads [18]:

$$
\frac{I_{1}(U)}{U I_{0}(U)}+\frac{n_{0}^{2}}{n_{p}^{2}} \frac{K_{1}(W)}{W K_{0}(W)}=0 .
$$

The effective index $n_{\text {eff }}$ is obtained by solving numerically the dispersion equation in the complex plane. The imaginary part of $n_{\text {eff }}$ gives the attenuation of the field during propagation. The pointing vector $S_{z}$ takes the form

$$
S_{z}=\frac{1}{4} c \varepsilon_{0}\left[E_{r} H_{\theta}^{*}+E_{r}^{*} H_{\theta}\right],
$$

and is used to calculate the radiated power and longitudinal electric field in the plasma with

$$
P_{z}=\iint S_{z} r d r d \theta
$$

Fig. 5 shows the calculated attenuation of the electric field component for two typical radii of the plasma. Here $v_{e}=15 \times 10^{12} \mathrm{~s}^{-1}$. The first case: $a=100 \mu \mathrm{m}$ models a single filament. The second case $a=450 \mu \mathrm{m}$ models a bundle of focused filaments and corresponds to the realistic case of our experimental conditions. The amplitude of the propagating microwave field calculated for a mm-diameter plasma is similar to the experimental curve of Fig. 4 and supports the hypothesis of a surface wave coupling. Assuming a perfect coupling for $a=450 \mu \mathrm{m}$, we find $n_{\text {eff }}=1.015-0.3203 \mathrm{i}$ and the magnitude of the longitudinal component of the electric field of the $T M_{0}$ mode at the interface is found to be $E_{\mathrm{z}}=1.2 \mathrm{kV} / \mathrm{cm}$ for $100 \mathrm{~W}$ of microwave power.

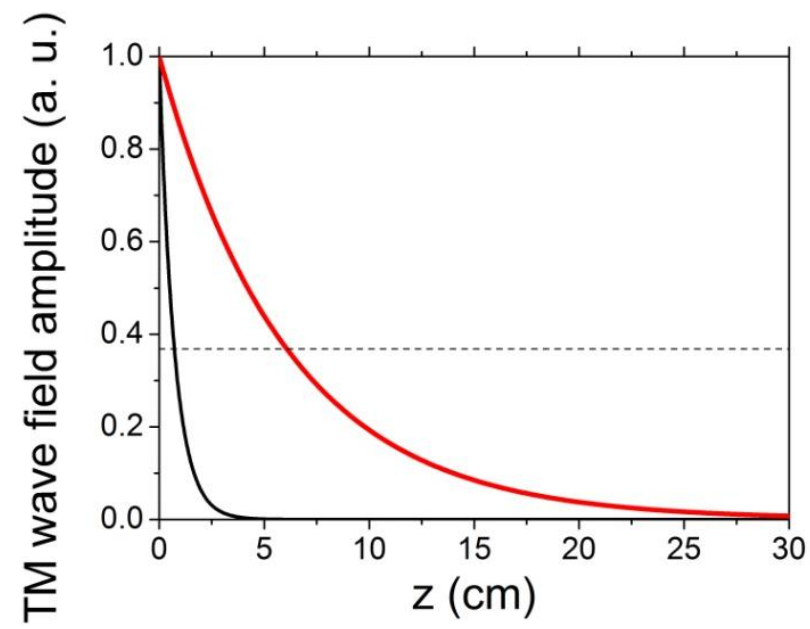

Fig. 5 Normalized amplitude of the electric field of the $T M$ surface wave as a function of propagation length calculated with: $n_{e}=10^{17} \mathrm{~cm}^{-3} ; n_{0}=1 ; v_{e}=15 \times 10^{12} \mathrm{~s}^{-1}$. In black: $a=100 \mu \mathrm{m}$ (attenuation length of $7 \mathrm{~mm}$ ). In red: $a=450 \mu \mathrm{m}$ (attenuation length of $6 \mathrm{~cm}$ ). The dashed horizontal line indicates the attenuation length defined at 1/e.

What is the fate of the amplified longitudinal current? As discussed now, it leads to the amplified emission of a characteristic short conical $\mathrm{THz}$ pulse by the plasma. This pulsed emission of a few ps duration is due to the heavily damped longitudinal free plasma oscillations [18]. It presents a peak at $1 \mathrm{THz}$ with a 3-THz bandwidth, ranging from low frequencies up to several THz. As shown in ref. [20], a $D C$ electric field leads to an amplification of this broadband THz emission. Since 
in the present case the duration of the laser pulse ( 100 fs) and of the damped longitudinal oscillations of the plasma (1-2 ps) are very short as compared to a microwave cycle $(\sim 1 \mathrm{~ns})$, the plasma can be viewed as under the influence of a constant electric field. We have measured the amplification of the $\mathrm{THz}$ emission through three spectral windows, around $3 \mathrm{GHz}, 12$ $\mathrm{GHz}$ and $91 \mathrm{GHz}$.

The I-dot detects the low frequency components of this amplified short THz pulse between 1 and 5 GHz. Fig. 6 shows the normalized spectra calculated by taking the Fourier transform of the signals of Fig. 3. Also shown is the injected microwave spectrum. To measure the microwave line, a small metallic wire was introduced in the surfatron along $z$ axis in the absence of filament. In this case, the microwave signal recorded is quasi monochromatic and centered at $2.45 \mathrm{GHz}$ (see violet curve). In the presence of filaments, the microwave signal covers the spectral bandwidth of the $I$-dot probe, as expected.

We have also detected an amplification of the transverse $\mathrm{THz}$ emission from the filament at $12 \mathrm{GHz}$ with a homodyne detector and at $91 \mathrm{GHz}$ with a selective heterodyne detector. By comparing the amplification of the signal obtained with the surfatron to the amplification of $\mathrm{THz}$ radiation in the presence of a longitudinal $D C$ field [20], one can estimate the magnitude of the longitudinal component of the electric microwave field acting on the plasma to be $\sim 1 \mathrm{kV} / \mathrm{cm}$ for an incident microwave power of $100 \mathrm{~W}$, in good agreement with the calculated value of the longitudinal component of the electric field at the interface between plasma and air.

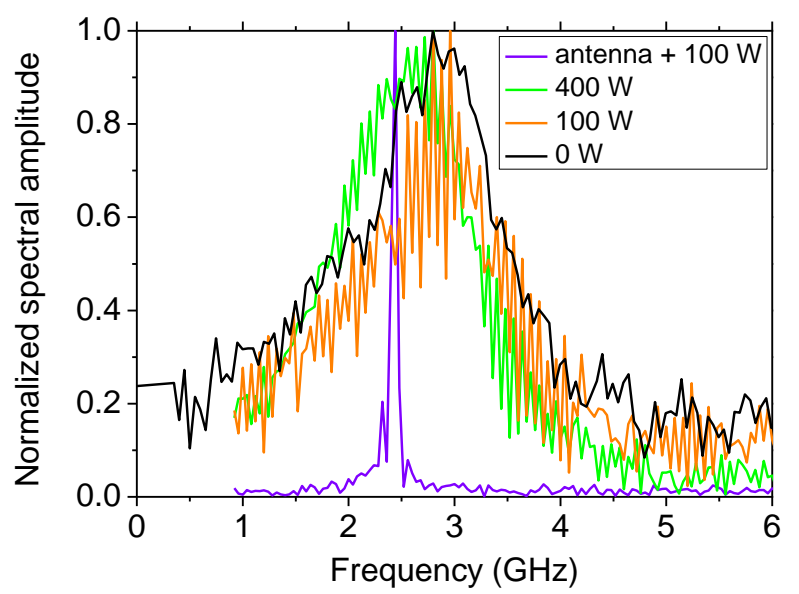

Fig. 6 Normalized spectral amplitude of longitudinal current as a function of frequency for different injected microwave powers.

It is instructive to evaluate the flow of excitation with other injected RF frequencies. In table I we show the calculated propagation distance for different frequencies and plasma diameters. As can be seen, the propagation distance is between one half and one third of the excitation wavelength, thus the excitation transfer distance covers several meters with $\mathrm{MHz}$ frequencies. With a sufficiently strong field amplitude $E \sim 15 \mathrm{kV} / \mathrm{cm}$, corresponding to a RF wave of a few tens of $\mathrm{kW}$ power, an avalanche regime would be reached, leading to a self-sustained virtual antenna [21]. An avalanche induced breakdown would lead to a high density hot plasma that would behave like a metallic antenna, as demonstrated in ref. [6].

TABLE I. Calculated attenuation length of the RF longitudinal electric field for various frequencies and plasma diameters.

\begin{tabular}{|c|c|c|c|}
\hline $\begin{array}{c}\text { Frequency } v \\
(\mathrm{GHz})\end{array}$ & $\begin{array}{c}\text { Wavelength } \lambda \\
(\mathrm{m})\end{array}$ & $\begin{array}{c}\text { Plasma radius } a \\
(\mathrm{~mm})\end{array}$ & $\begin{array}{c}\text { Attenuation length } L \\
(\mathrm{~m})\end{array}$ \\
\hline 2.45 & 0.122 & 0.45 & 0.06 \\
\hline 0.245 & 1.22 & 1.3 & 0.66 \\
\hline 0.0245 & 12.2 & 3 & 4 \\
\hline
\end{tabular}

In conclusion, we have shown evidence of the coupling of microwave radiation to a multi-filament plasma column and its propagation out of the excited region of the plasma in the form of a $T M$ wave. This leads to the amplification of longitudinal oscillations of the plasma over a distance exceeding $10 \mathrm{~cm}$. We predict that this transfer could reach several meters if lower frequency is injected in a larger plasma radius.

\section{Acknowledgments}

This project has been supported by the French Direction Generale de l'Armement (Grant $\mathrm{N}^{\circ} 2013.95 .0901$ and $\mathrm{N}^{\circ}$ 2009.34.0031). The authors acknowledge technical support by Yves-Bernard André, Jérôme Carbonnel, Guillaume Point, Bruno Honnorat and fruitful discussions with Sylvain Flajolet and Besma Larbi. 


\section{References}

1. A. Couairon, and A. Mysyrowicz, Phys. Rep. 441, 47 (2007)

2. C. D'Amico, A. Houard, M. Franco, B. Prade, A. Mysyrowicz, A. Couairon, and V.T. Tikhonchuk, Phys. Rev. Lett. 98, 235002 (2007)

3. L. Arantchouk, A. Houard, Y. Brelet, J. Carbonnel, J. Larour, Y.-B. André and A. Mysyrowicz, Appl. Phys. Lett. 102, 163502 (2013)

4. M. Rodriguez, R. Sauerbrey, H. Wille, L. Wöste, T. Fujii, Y.-B. André, A. Mysyrowicz, L. Klingbeil, K. Rethmeier, W. Kalkner, J. Kasparian, E. Salmon, J. Yu, and J.-P. Wolf, Opt. Lett. 27, 772 (2002)

5. A. Houard, C. D’Amico, Y. Liu, Y. B. André, M. Franco, B. Prade, A. Mysyrowicz, E. Salmon, P. Pierlot, L.-M. Cléon, Appl. Phys. Lett. 90, 171501 (2007)

6. Y. Brelet, A. Houard, G. Point, B. Prade, L. Arantchouk, J. Carbonnel, Y.-B. André, M. Pellet, A. Mysyrowicz, Appl. Phys. Lett. 101, 264106 (2012)

7. L. A. Johnson and P. Sprangle, J. Appl. Phys. 118, 123301 (2015); P.-Q. Elias, Journal Aerospace Lab, Special Issue 10, $03(2015)$

8. M. Alshershby, J. Lin, Z. Hao, Appl. Phys. B 108, 859 (2012)

9. V.P. Kandidov, S.A. Shlenov, O.G. Kosareva, Quantum Electronics 39, 205 (2009)

10. R. Musin, M.N. Shneider, A.M. Zheltikov, and R. B. Miles, Applied Optics 46, 5593 (2007)

11. M. N. Shneider, A.M. Zheltikov, and R. B. Miles, J. Appl. Phys. 108, 033113 (2010)

12. M. Alshershby, J. Lin and Z. Hao, J. Phys. D: Appl. Phys. 45, 065102 (2012)

13. M. Châteauneuf, S. Payeur, J. Dubois and J.-C. Kieffer, Appl. Phys. Lett. 92, 091104 (2008)

14. N. A. Bogatov, A.I. Kuznetsov, A.I. Smirnov, A.N. Stepanov, Quantum Electronics 39, 985 (2009)

15. V.D. Zvorykin, A.O. Levchenko, A. V. Shutov, E.V. Solomina, N.N. Ustinovskii, and I.V. Smetanin, Phys. Plasmas 19, 033509 (2012)

16. G. Point, Y. Brelet, A. Houard, V. Jukna, C. Milián, J. Carbonnel, Y. Liu, A. Couairon, and A. Mysyrowicz, Phys. Rev. Lett. 112, 223902 (2014)

17. M. Moisan and Z. Zakrzewski, J. Phys. D: Appl. Phys. 24, 1025 (1991)

18. B. Zhou, A. Houard, Y. Liu, B. Prade, A. Mysyrowicz, A. Couairon, P. Mora, C. Smeenk, L. Arissian, and P. Corkum, Phys. Rev. Lett. 106, 255002 (2011)

19. H. Nowakoska, Z. Zakrzewski and M. Moisan, J. Phys. D: Appl. Phys. 34, 1474 (2001)

20. Y. Liu, A. Houard, B. Prade, A. Mysyrowicz, A. Diaw, and V.T. Tikhonchuk, Appl. Phys. Lett. 93, 051108 (2008)

21. S. B. Bodrov, D.I. Kulagin, Yu A. Malkov, A. A. Murzanev, A.I. Smirnov and A.N. Stepanov, J. Phys. D: Appl. Phys. 45, 045202 (2012) 